\title{
Association of Apolipoprotein E Gene Polymorphism with Lipid Profile in Patients with Acute Coronary Syndrome in Han Chinese: A Critical Review
}

\author{
Abdul Qadir Nawabi', Mingming Yang1, Xiangming Cai'2, Wenjing Gou², Jiantong Hou', \\ Veeda Nizami ${ }^{3}$, Qiming Dai ${ }^{*}$ \\ ${ }^{1}$ Department of Cardiology, The Affiliated Zhongda Hospital, Southeast University, Nanjing, China \\ ${ }^{2}$ College of Medicine, Southeast University, Nanjing, China \\ ${ }^{3}$ Dalian Medical University, Dalian, China \\ The Corresponding author is entitled to Qiming Dail*,Email: 20941705@qq.com
}

How to cite this paper: Nawabi, A.Q., Yang, M.M., Cai, X.M., Gou, W.J., Hou, J.T., Nizami, V. and Dai, Q.M. (2019) Association of Apolipoprotein E Gene Polymorphism with Lipid Profile in Patients with Acute Coronary Syndrome in Han Chinese: A Critical Review. World Journal of Cardiovascular Diseases, 9, 825-845. https://doi.org/10.4236/wjcd.2019.911074

Received: May 24, 2019

Accepted: November 17, 2019

Published: November 20, 2019

Copyright $\odot 2019$ by author(s) and Scientific Research Publishing Inc. This work is licensed under the Creative Commons Attribution International License (CC BY 4.0).

http://creativecommons.org/licenses/by/4.0/

cc) (7) Open Access

\begin{abstract}
This review paper focuses on the genetic contribution, in particular, the association of Apolipoprotein E gene polymorphism to lipid abnormality and subsequent acute coronary syndrome in Han Chinese of China. Many researches have been published pertaining the influence of ApoE gene polymorphism on coronary artery disease, dyslipidemia and the response of statin in Han Chinese. Most of the studies in Han Chinese like other populations demonstrated that ApoE 4 allele genetically predisposes coronary artery disease, acute coronary syndrome, severity of occlusion of coronary artery and higher incidence of major adverse cardiovascular events (In Han Chinese, ApoE allele carriers demonstrated $85 \%$ increase in major adverse cardiovascular events (MACE) in six months follow up). In addition, ApoE4 allele carrier also showed both increased in LDL level and decrease response to statin therapy in dyslipidemic Han Chinese. On the other hand, ApoE2 carrier is scavenger of cholesterol and triglyceride from the blood; thus it is cardiovascular-protective. Despite positive relationship between ApoE gene polymorphism and cardiovascular pathologies, prognostic outcome and resistance to intervention, this area of research still requires extensive investigation in Han Chinese. Because, several other studies revealed either negative effect or showed no effect by ApoE gene polymorphism on cardiovascular disease. Some of the causes of such debatable results could be explained by factors such as diminutive frequency allele and expression of ApoE gene in coronary heart disease. This part of the research yet requires extensive study with bulkier sample size and retrospective in nature, in order to ascertain the influ-
\end{abstract}


ence of ApoE genotype on lipid, anti-hyperlipidemic agent and coronary heart disease. Such studies could assist us to confirm whether to test healthier subjects to predict genetic risk of coronary heart disease in Han Chinese population. The aim of this review paper is to critically analyze the effect of ApoE gene on the occurrence of coronary heart disease in Han Chinese.

\section{Keywords}

Apolipoprotein E Gene Polymorphism, Acute Coronary Syndrome, Dyslipidemia, Han Chinese

\section{Introduction}

Among adult cardiovascular mortality in China, Coronary Artery Disease stands as $2^{\text {nd }}$ prominent cause [1] [2]. Although the prevalence of Coronary Artery Disease keeps rising in China, its prevalence is comparatively low to western countries. The data released in 2015 by China Health and Family Planning Commission's Statistical yearbook demonstrated that the death rate from CAD has drastically ascended in China as shown in Figure 1 [3]. There is a continuous tendency of increased mortality and morbidity from Coronary Artery Disease in urban and rural areas. However, it is dramatically greater in metropolitan areas than country areas. Some of the factors of higher mortality and morbidity caused by urbanization are unhealthy life style and increased number of old age population [4].

Presently, the most common etiology of both mortality and morbidity in China, is led by acute coronary syndrome, which has approximately reached 2.8 million Syndrome patients in China [1]. The rapid transition in life style in China and concomitant intensification in major risk factors have resulted in high incidence of ACS admissions in hospitals as shown in Figure 2 [5], as it can be observed in the figure that morbidity and mortality in both urban and rural areas have drastically augmented.

Coronary Artery Disease is a poly-factorial sickness, which includes psychological factors, socioeconomic status, life style and genetic factors. Classical risk factors such as diabetes mellitus, HTN, dyslipidemia and smoking account for 85 to 90 percent of coronary heart disease [5] [6].

On the contrary, there are $10 \%$ to $15 \%$ patients who don't carry classical risk factors, and however, get coronary heart Disease. Some of them could be attributed to genetic cause, in particular premature Coronary Artery Disease which could be related to genetic component. We need to concentrate on newer factors in order to be able to forecast the future risk and determine timely intervention in addition to the conventional risk factors as genetic factors, in particular gene polymorphism [7].

The knowledge of genes affecting cardiovascular diseases, is very limited. Most of the cardiovascular diseases are polygenetic. We know little about cardiovascular 


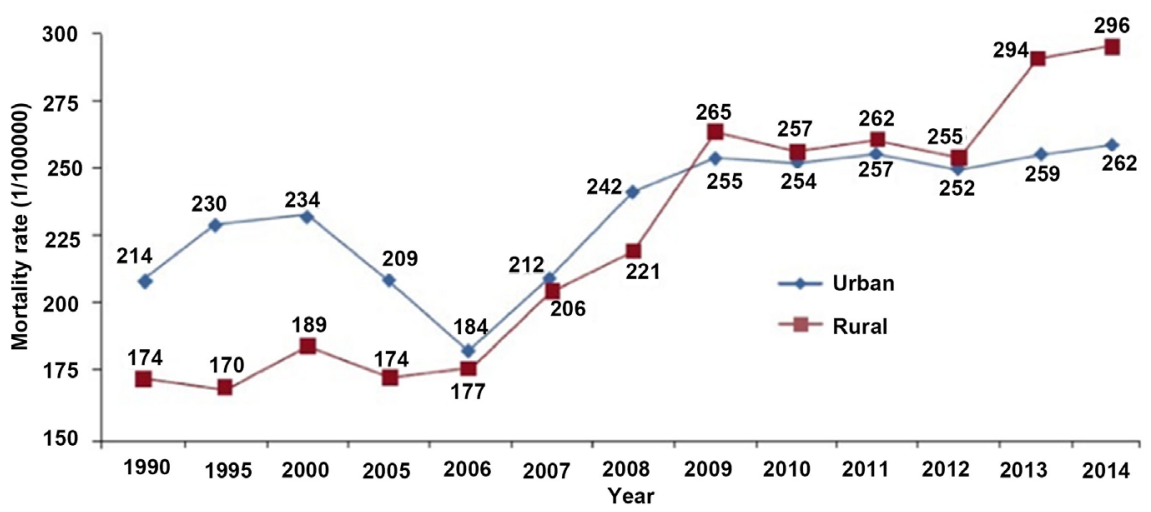

Figure 1. Coronary heart disease mortality tendency in city and country-areas in China: 2002-2014. Exported from Wei-Wei CHEN, et al. [1].

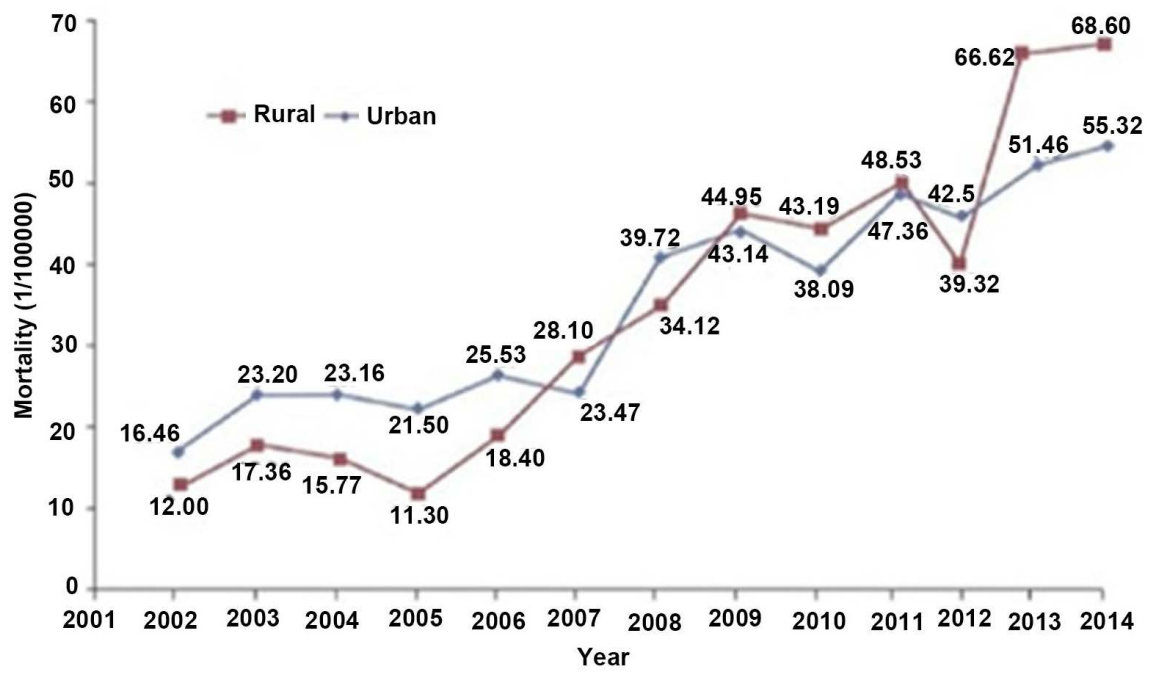

Figure 2. AMI mortality tendency in urban and rural regions in China: 2002-2014. Exported from Wei-Wei CHEN, et al. [1].

disorders which are single-gene Mendelian pattern. Examples of such disorders are familial hypercholesterolemia, long QT syndrome and hypertrophic cardiomyopathy [8].

Familial hypercholesterolemia which is a single gene lipid disorder, can cause hyperlipidemia and subsequent premature Coronary Heart Disease [9]. Apolipoprotein $\mathrm{E}$ gene is a highly investigated and the most intriguing gene, which is polymorphic in different ethnic groups; however there isn't any confirmed clinical implication.

The impending genetic threatening factor, is ApoE gene polymorphism for coronary artery disease and acute coronary syndrome. Structurally, human ApoE is a glycoprotein, made up of 299 amino acids, found as an important component of different lipoprotein such as HDL, intermediate density lipoprotein, very low density lipoprotein and chylomicrons L. It works as a ligand to help in the uptake of lipid and bind to the respective receptor, thus scavenging lipid from the serum [10]. 
ApoE protein, is the product of the ApoE gene which is situated on chromosome 19q 13.2, consists of three introns and four exons [11] [12]. It is consists of 3597 nucleotides. ApoE polymorphism has three different isoforms which are ApoE2, ApoE3 and ApoE4, synthesizing six various alleles such as E2/E2 (counted as E2 or E2 homozygous), E2/E3 (counted as E2 or E2 carrier), E3/E3 (counted as $\mathrm{E} 3$ and is the most common isoform amongst all three isoforms), E2/E4, E3/E4 (counted as E4 carrier), and E4/E4 (E4 homozygous) [13].

Since ApoE genotype polymorphism was first defined in patients who had dysbetalipoproteinemia and the effect of ApoE gene polymorphism that is why multiple studies were piloted to find the influence of lipid and subsequently cause coronary artery disease in various racial group. Since it is polymorphic in nature, which means there is variation amongst the expression of particular isoform, thus different prevalence of cardiovascular diseases in different ethnicities.

The polymorphic nature of the gene explains the mechanism by which altered phenotype changes the lipid metabolism, thus either leading the pathway for atherosclerosis or protecting against it [8] [14]. In most of reports amongst different populations, it has been documented that ApoE4 isoform predisposes to genetically higher risk of acquiring coronary heart disease and acute coronary syndrome while ApoE2 carrier has a negative linkage with CHD. In addition, people who have longer life time, are carrier of ApoE genotype.

Song y et al. reported the first meta-analysis on this particular gene polymorphism and ischemic heart disease, the result was expected as ApoE allele, with greater chance of acquiring IHD in contrast to E3 homozygous patients. In similar study, it was reported E2 carrier had no impact on ischemic heart disease (OR, 0.98 [CrI, 0.66 to 1.46] [15]). Nevertheless, different factors were not considered into account such as variation in topography, gender, allele frequency in dissimilar populations, which made is less reliable. In addition, since the frequency of genotype varies from population to population, ApoE gene from various ethnic groups cannot be meta-analyzed. For example, a study performed in Afro-Caribbeans did not show any high difference in Acute Coronary Syndrome and ApoE E genotype. However, it did show that ApoE4 had hyperlipidemia [16]. This contrasts with other studies both in Han Chinese and other racial groups, which show a positive relationship with ApoE4 and Coronary Artery Disease.

In contrast to other Asians such as Japanese (10.5\%), Korean (9\%), Thai (12.54\%), Malaysian, and other ethnicities, Han Chinese (7.5\%) makes the lowest frequency of ApoE4 in Asia [17]. Because of the genetic difference in ApoE E gene, recently, several basic researches and cross-sectional researches have been engrossed on the impact of ApoE gene polymorphism and ischemic heart disease amongst Han Chinese. However, the results have been inconsistent and many studies have been published in Chinese language. In addition the samples of most the studies are small and cross-sectional. Therefore a study with adequate sample size is required. This review would focus on the relationship of ApoE gene polymorphism on Coronary Artery Disease in Han Chinese, specially, Acute Coronary Syndrome, the effect of statins in different alleles of ApoE 
polymorphism and the mechanism of ApoE gene impacting as a genetic risk factor in the pathophysiology of ischemic heart disease.

\section{Apolipoprotein E Isoform and Its Influence on Lipid Metabolism}

ApoE was for the first time known in patients with dysbetalipoproteinemia and its contribution in lipid metabolism is one of the highly postulated mechanisms causing dyslipidemia and secondarily leading to Coronary Artery Disease.

In order to understand the effect of Apolipoprotein isoform on lipid metabolism, one must know the normal morphology of ApoE phenotypes and its interaction with the receptor. Studies have been published which have shown that some of the isoforms of Apolipoprotein E are related to cardiovascular diseases while others are protective. It solely depends which isoform is present in a subject type of ApoE isoform.

\subsection{Structure of Apolipoprotein Isoform}

As shown in Figure 3 Apolipoprotein E protein is made up of two peculiar anatomical and functional parts which are hinged by consisting of two eccentrically structural and functional domains that are interlocked by an enzyme sensitive protease-sensitive coil [18]. The carboxyl-terminal domain contains sites for both lipid and heparin [18] [19]. These sites physiologically on c-term are believed to perform a vital role, by facilitating the collaboration between the lipid particles and cell-surface heparin sulfate proteoglycans on the vascular wall, augmenting the catalytic function of LPL leading to hydrolysis of these lipid particles [19].

On the other hand, $\mathrm{NH}_{2}$ end of the protein is supposed to bind to the various receptors. ApoE performs as a ligand for multiple hepatic receptor in order to clear metabolic remnants of Apolipoprotein B-containing lipoprotein, particularly chylomicrons and VLDL, which traffic triglycerides from the intestine and liver to the peripheral tissue respectively [18]. The receptors which bind to $\mathrm{NH}_{2}$ terminus of the ApoE are VLDL receptor, LDL receptor and LDL receptor-related proteins through heparin sulfate proteoglycan(HSPG) [21] [22] (Figure 4).

\subsection{Dispersal of ApoE on Lipoproteins}

The lipoprotein pathway and the involvement of Apolipoprotein $\mathrm{E}$ has been demonstrated in Figure 5. There is no ApoE isoform present on IDL and LDL lipoprotein in pre-prandial state but found on VLDL and HDL. After food intake, the residues of chylomicrons like that of VLDL are sequestered swiftly. Both the ApoCii and ApoE are present in 50\% of the VLDL [23]. The co-existence of ApoE with ApoCii, makes the clearance of lipid stalled which is facilitated via ApoE. The lipo B-holding lipoprotein comprising ApoE which contain ApoE doubles in quantity in 4 to 5 hours post-prandially, and slowly regresses to LDL after six to 7 hours and at 10 hours completely vanishes [24].

The synthesis of HDL is started by ApoE with the help of ABCA1 transporter by transporting cholesterol and phospholipid from the ABCA1 transporter, 


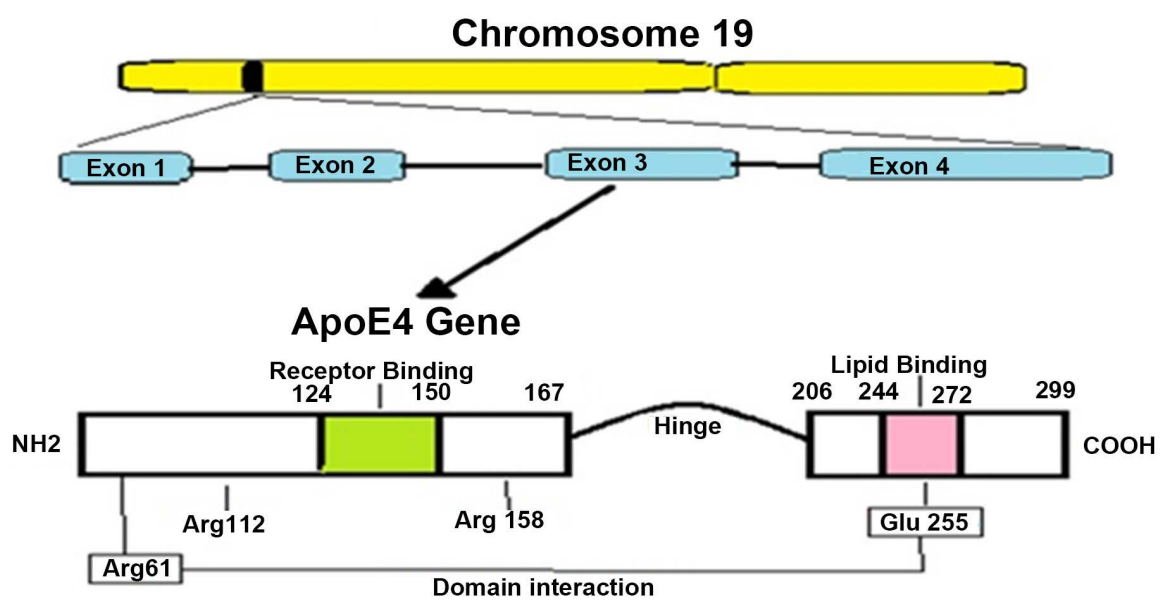

Figure 3. Showing the structure of Apolipoprotein E (The figure has been modified from "Localization of genes encoding apolipoproteins CI, CII, and E to the p 13-cen region of human chromosome 19. Scott J, Knott TJ, Shaw DJ, Brook JD” [11]).

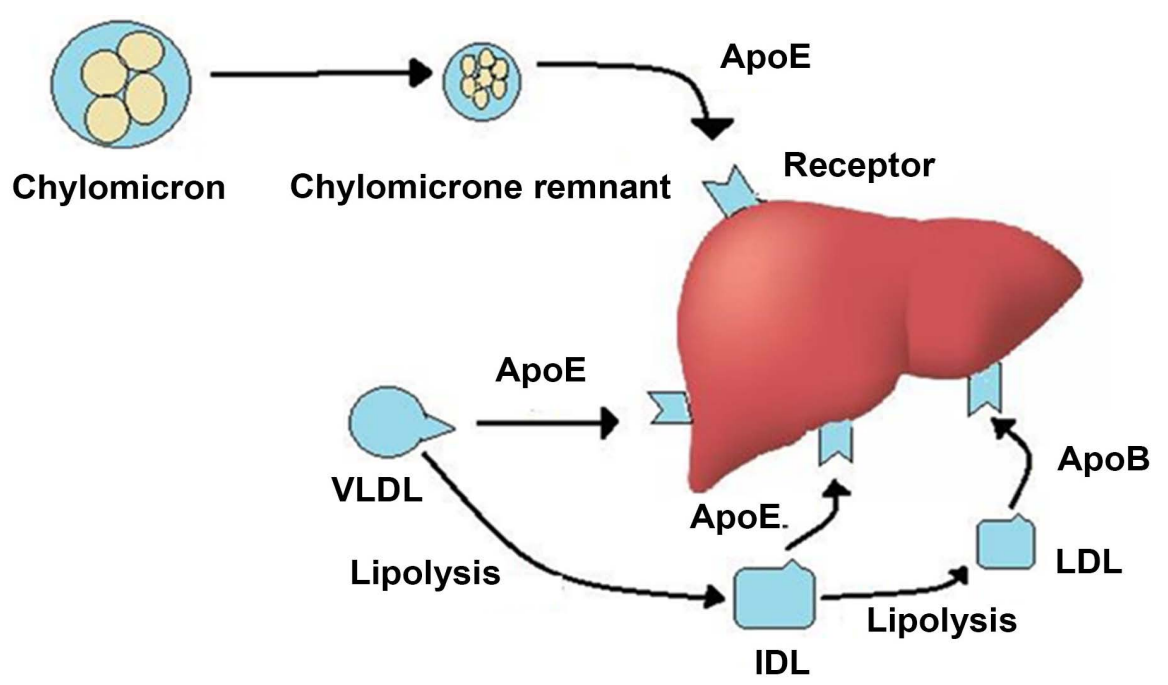

Figure 4. Shows the lipoprotein metabolism facilitated by diet derived chylomicrons and lipoprotein produced in the liver, VLDL are hydrolyzed in the circulation by lipoprotein lipase. Plasma lipoprotein metabolism mediated by ApoE. The residuals of chylomicron and remnants of VLDL can be cleared from the plasma with the help of liver, which has receptors for LDL or the HSPG/LRP pathway. Eventually, thoroughly hydrolyzed LDL have consumed the ApoE ligands and can merely the uptake of the LDL can be via Apolipoprotein $b$ (the figure is modified from Division of Translational Medicine and Human Genetics, Perelman School of Medicine at the University of Pennsylvania, 11 - 130 Translational Research Center, Philadelphia, PA, USA [20]).

however, there is variation in effective function in the isoform of ApoE. For example ApoE3 is a better synthesizer of HDL than ApoE4. The efficiency of ApoE4 is upgraded by peptide CS 6253 [25].

\subsection{Effect of ApoE Isoforms on Lipoprotein}

It has been shown that different isoforms of ApoE have different effect on different Apolipoprotein. For instance, it was revealed by M S Weintraub et al. that 


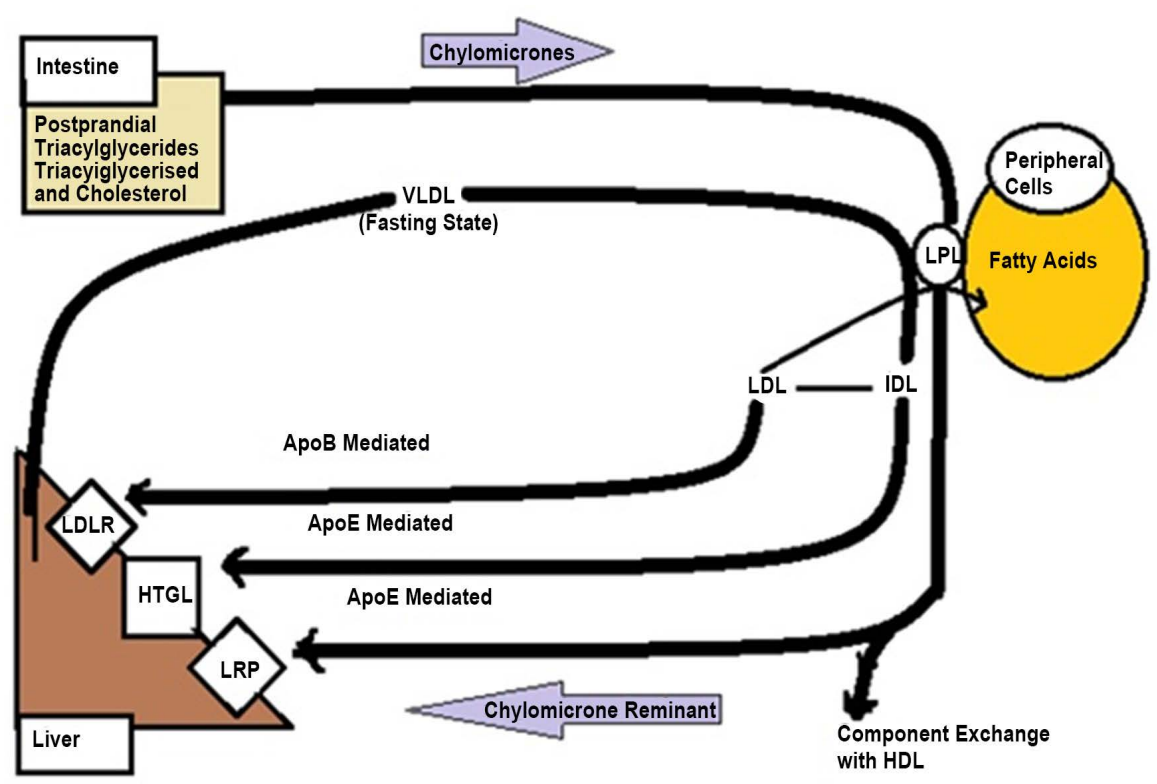

Figure 5. The lipoprotein pathway, metabolism and the involvement of Apolipoprotein E.

the clearance of chylomicron was delayed by ApoE2 isoform while was speedy with ApoE3 and ApoE4 isoforms [26]. In a similar manner, it was also demonstrated that ApoE genotype had noticeable influence on ApoE genotype attached to VLDL but null effect on the free plasma ApoE isoforms. ApoE protein is discovered in different sequences on VLDL particles, however, it is at low amount in people with ApoE4, which is explains increased clearance [27].

ApoE isoform mostly influence LDL and apolipoprotein B plasma concentration in fasting state. The effect also depends on sex and premenopausal and postmenopausal state of females, which clearly shows hormonal influence. For example in male, ApoE2 decreased LDL cholesterol by $0.24 \mathrm{mmol} / \mathrm{L}$ while in female subjects, it was between $0.21 \mathrm{mmol} / \mathrm{L}$ to $0.53 \mathrm{mmol} / \mathrm{L}$ depending on premenopausal and postmenopausal state, respectively [28]. On one hand, ApoE isoform is not present on LDL, on the other hand it affects the size and the plasma quantity of LDL.

It is known that minute LDL is associated with ischemic heart disease and decrease level of HDL. There are many researches which have shown that ApoE isoform have various effect on LDL and HDL, however, both methodology and participants were different. ApoE2 carrier showed minute LDL particle while no effect on the size of HDL particle, on the other hand, ApoE4 carrier showed smaller size of HDL particle but no effect on LDL particle size [29]. A study conducted in intermediate aged Curation patients, showed ApoE2 carrier had effect on the size of LDL particle in male while no effect in female [30].

Another study in Kuwaiti Arabs reported that there was no difference on LDL particle size between ApoE2 carrier and non-ApoE2 healthy subjects. However, elderly subjects carrying ApoE2 with coronary artery disease had smaller particle 
of LDL [31].

ApoE protein found on the subset of Apolipoprotein A. In lipoprotein, which have $A p o E$ in its structure, should also consist $A p o(A)$ and $A p o(B)$ in minute size almost like LDL as in contrast to the found on VLDL. Lipoprotein (A) which is of two types, which is classified based on the presence and absence of ApoE. Bach-Ngohou $\mathrm{K}$ et al. in normal lipid profile subjects showed almost 16.2\% ApoE [Lp(a):B:E] and high triglyceride. In this study, ApoE did not have any direct effect on ApoE [Lp(a):B:E]. However, ApoE attached to [Lp(a):B:E] plays an important role in the concentration of lipoprotein a subtype and subsequently triglyceride-dominant particles [32].

ApoE gene polymorphism affects the Apolipoprotein A clearance and plasma concentration. For example, ApoE2/E2 genotype has $65 \%$ less concentration of Apo(A) than ApoE4/E4 genotype [33]. In addition, elevated plasma concentration of $\operatorname{Apo}(\mathrm{A})$ also showed elevated LDL amount which is well-matched to familial hypercholesterolemia (concluding that there is little activity at the LDL receptor level). E2/E2 did not have too much effect on Apo(A) plasma amount as it was anticipated. However, it augmented the increment of Apo(A) in subjects with compromised plasma clearance of LDL. ApoE2 genotype is a very robust factor in determining the low concentration of Apo(A). Nevertheless, it did not alter the relationship of Coronary Artery Disease and Aortic Stenosis with Apo(A).

A study by Moreno JA et al. reported that both diet and ApoE genotype affect the size of lipoprotein [34]. According to them individuals with ApoE4 carriers had bigger size of LDL. Subjects with ApoE4 carriers who were subjected to sugar-rich diet, had enlarged LDL, the exact mechanism of increased VLDL synthesis would be credited to the fatty acid oleic acid causing high triglyceride which refashioned the more slowly revolving LDL particle. In this research it was also reported that ApoE carrier had tinier LDL particle size. These smaller LDL particles do not depend on the size.

Sunny H. Zhang, et al. demonstrated that the knockout mice of Apolipoprotein E gene had higher level of cholesterol and subsequently after 8 months contracted coronary artery lesion as compare to wild mice [35]. The work by Sunny H. Zhang, et al. proved that the functional integrity of Apolipoprotein is very essential for the sequestration of lipid and subsequently protecting against dyslipidemia.

The isoforms of ApoE simply varies from one another by substituting two amino acids at two positions, however, the isoforms are rendered drastic change both in term of chemistry and physiology, which in turn would the lipid metabolism and subsequently induce cardiovascular pathology. Numerous studies in different ethnicities have shown that ApoE4 genotype is greatly related with dyslipidemia while ApoE2 is proved to be protective [36] [37] [38] [39] [40]. However, according to the study by Larifla Li et al. in 712 Afro-Caribbean healthy and subjects with Coronary Artery Disease did not show any relationship be- 
tween Apo 4 and hyperlipidemia $(\mathrm{p}=0.002)$ [16].

On the other hand, it has been studied in great detail that interactions between Apo-E attached to lipoprotein and LDL receptor are very significant for hyperlipidemia and CVD. For instance, Versmissen $J$ et al. revealed that in patients homozygous for ApoE4, having mutated LDL receptor, had lower risk of Coronary Artery Disease (hazard ratio, 0.16; 95\% CI, 0.05 - 0.58; p = 0.005) [41]

\section{ApoE Polymorphism and Its Impact on Blood Lipid Level in Han Chinese}

The China Health and Nutrition in 2012 reported a survey on the total prevalence of lipid in Chinese population was as follows: total cholesterol (TC) $\geq 6.22$ $\mathrm{mmol} / \mathrm{L}$ in males and females age above 18 were 4.7 and $5.11 \%$, respectively, and the frequency rates of triglyceride (TG) $\geq 2.26 \mathrm{mmol} / \mathrm{L}$ amongst males and females were $16.7 \%$ and $9.8 \%$, respectively [42]. Gan et al. showed in his prospective study amongst Han Chinese revealed that aging and menopause are the contributory factors of hyperlipidemia [42].

In addition to other acquired risk factors, there are multiple genes which have been elucidated in detail in different ethnicities including Han Chinese of China. The common genes which are responsible for hyperlipidemia and familial dyslipidemia are ApoE, LDLR, APOB and PCSK9 [43]. ApoE gene polymorphism and its relationship to lipid metabolism and the effect of anti-lipid therapy has been studied in Han Chinese. However, there are different results without confirmatory effects which can lead us for future therapy or can guide us in evidence based medicine. Since almost $90 \%$ of Chinese population comprises of Han ethnicity and approximately makes $1 / 5^{\text {th }}$ of human race [44] therefore, any study regarding gene and polymorphism in such a huge mono-ethnic population would be more credible and less biased.

The possible associations in humans between the ApoE genotype and the blood lipid level and familial hyperlipidemia in different ethnicities of the world have been evaluated in multiple studies [45] [46]. Yongjing Xia et al. in 2000 conducted a study in Han Chinese from two different healthy populations of Shanghai ( $\mathrm{n}=99$ ) and Beijing [47]. There was no significant difference in ApoE allele frequency in between the two populations, however, there was significant different in their phenotype and blood serum lipid [47]. The study proved that genetic polymorphism is not solely counting for hyperlipidemia [42]. On the other the study could be biased since the population size was very small.

A similar study was conducted in 2016 in Han Chinese of Shandong by ShuYi Han et al. was consistent with other studies, ApoE2 carriers have lesser amount of blood total cholesterol, LDL-C and Apo-B than ApoE3 carrier and of serum TC,LDL-C, and Apo-B than ApoE3 carriers and superior levels in ApoE4 carriers in comparison to ApoE levels. There have been numerous researches piloted, however, without any clinical orientation. A proper study with prospective study needs to be done in order to approach more clinical targeted and used for the 
betterment of patient.

\section{The Response of Statin in Han Chinese with ApoE4 Allele}

Published studies have proved the strong relationships between LDL-C and Coronary Artery Disease (Ference et al., 2017; Sampson, Fazio, \& Linton, 2012). Thus, Statins are one of the most broadly used cardiovascular medication, used for both treatment and prevention of cardiovascular diseases. It was emphasized by 2013 Chinese consensus that all the ACS patients should be administered with Statins irrespective to the level of lipid in the plasma and continue Statin even time-honored after the patient discharges (Schiele, Farnier, Krempf, Bruckert, \& Ferrieres, 2018). However, it was displayed by Zhang W et al. in his prospective study in ACS patients taking Statins and followed for one year, 48\% patients did not reach the target level of LDL (W. Zhang, Ji, Yu, \& Wang, 2017). There has been inter-individual discrepancy in pharmacological response and as a result change the clinical outcome [48] [49]. There are numerous factors which are responsible for this inter-individual inconsistency such as patient's compliance to the medication, health status of the patient, quality of medication, stage and prognosis of the pathology and the most important but neglected factor, the genetic factor.

There are many factors involved in the non-responsiveness of statin such as drug non-compliance to medication, intolerance to Statins because of adverse effects, and individual differences in response [50]. The individual differences in response can be polymorphism of genes, related to different genes responsible for lipid metabolism.

One of the most pharmacodynamically essential genes, is the ApoE polymorphic genes, which is vital in the metabolism of lipid. Many researches have been conducted on it, however, with erratic outcomes. A meta-analysis conducted in 2009 about ApoE gene polymorphisms and impact on response to statin therapy, concluded that there is no influence of ApoE gene polymorphism on Statin pharmacology [51]. However, this meta-analysis had multiple drawbacks, such as, comparison of studies between different ethnicities, use of different types of Statins in various studies, and the most important, different age groups. Kirac D et al. their study disclosed that patients with ApoE4, allele who were treated with Statins were less-responders as compared to ApoE2 while ApoE2 allele had higher level of triglyceride [52]. The same trend was seen in Chinese Han patients having dyslipidemia, treated with Rosuvastatin. However the study was not statistically significant [53].

A similar type of study was conducted in Kaifeng city of China, nevertheless, in a bigger sample size, showed decreased responsiveness to Atorvastatin in patients with ApoE4. This study also showed less expression of both ApoE3/ApoE3 and ApoE3/ApoE4 but the impact on the expression of ApoE3/E3 was more obvious. This study was published in Chinese language [54]. These above mentioned studies contrast to the study, which was done in Singaporean Chinese, 
reporting that ApoE3/ApoE4 have had better pharmacodynamics response to Simvastatin and Lovastatin than ApoE3/E3 [55].

The exact mechanism of ApoE isoform influence on lipid profile and response to statin therapy is related with the pattern of amino acid in ApoE isoforms. For instance, ApoE2 has less than 2\% affinity in contrast to other isoforms but low lipid profile and low incidence of coronary artery disease. This is indorsed to the compensatory increment of receptor LDL-R. On the other hand, ApoE4 which attaches favorably to the triglyceride-predominant VLDL, as a result assists in speeding the clearance the VLDL particles from the plasma. This phenomenon consequences into down-regulation of the LDL-R resulting in hyperlipidemia [56]. This mechanism is further elucidated by many studies in familial hypercholesterolemia patients, who have degradation of the LDL-R. These studies have demonstrated that it is not the direct ApoE isoforms important in the response to Statin but rather the receptors of LDL-R and ApoE isoforms, which are essential in the response of statin therapy.

China is a big country, with different cuisines and slightly different life style, it would not be tranquil to stipulate the effect of one polymorphic gene on lipid profile and ApoE gene affecting the effect of Statin in just a small sample size selected. Similarly in Han population, there is different effect of ApoE on lipid profile. For instance, both the genotypes and the lipid profile were different in two different subjects of Shanghai [47]. On the other hand, numerous fragmented studies have evidenced that apolipoprotein e4 allele may have been one of the neglected potential causes for non-respond of dyslipidemia in patients treated with statin and consequently may affect the treatment plan of ischemic heart disease. Nonetheless, it is still very premature to adopt with confirmation that ApoE genotype polymorphism would affect the clinical outcome in ischemic heart disease, particularly acute coronary syndrome. Further study needs to be done in Han Chinese, with large number of sample size and with the same class of statins in order to confirm the impact of ApoE gene on the therapeutic effect of stain in coronary artery disease.

\section{The Relationship of ApoE Gene Polymorphism to Acute Coronary Syndrome in Han Chinese}

There are multiple case-control studies conducted concerning the ApoE genotype and Acute Coronary Syndrome in various ethnicities. Different parts of the world have displayed the relationship between ApoE genotype polymorphism and Acute Coronary Syndrome [57]-[62]. It is generally observed from these researches that ApoE2 allele is known to be protective for Coronary Artery Disease and Acute Coronary Syndrome while ApoE 4 allele is an alleged to be risk factor for Ischemic Heart Disease. However, relationship between ApoE gene polymorphism and Coronary Artery Disease has been inconsistent. For example in a study on in Afro-Caribbean subjects with CAD, there was a negative correlation between ApoE4 and Acute Coronary Artery Disease but one has to carefully ana- 
lyze the conclusion since the study was with insignificant sample size, making it susceptible to more biased outcome [16].

Han Chinese which is making the largest ethnic group, counts for one fifth of world population and Han Chinese are making 90\% of population of China [63]. Any study regarding ApoE genotype in such a large ethnic group would be more reliable and would be less confounding (Table 1).

As far as the frequency of ApoE genotype in Han Chinese is concerned, it is as following. $\mathrm{E} 3 / 3$ is $75 \%, \mathrm{E} 3 / 4$ is $10.7 \%$ and $\mathrm{E} 2 / 3$ while $0.60 \%, 1.20 \%$, and $0.60 \%$ for E2/2, E2/4, and E4 [64]. However, the frequency of the ApoE genotype may vary from one demographic region to another, even in China in same ethnic group the frequency may vary widely. Numerous cross sectional studies and 2 meta-analysis have been conducted unto now. Both meta-analysis show positive relationship between Coronary Artery Disease and Ischemic Heart Disease [65]. Most of these studies have been conducted in Han Chinese, though with small sample size. They show inconsistent result. There are two meta-analysis recently done in Han Chinese, which shows that ApoE4 allele is one of the genetic factors associated with Coronary Artery Disease, in turn with Acute Coronary Syndrome [60] [62] [66]. These studies have only mentioned regarding Coronary Artery Disease and have not specified ACS. There is only limited number of studies in relationship to ApoE gene polymorphism and Acute Coronary Syndrome in Han Chinese [67] [68]. Bai and colleagues reported their study in Chinese while Baum et al. did not conducted their research in the mainland of China.

In order to get authenticity and confirmation of ApoE genotype relationship to Acute Coronary Syndrome, one has to be vigilant regarding the interpretation of the results the studies. We need further study with a bigger sample size or meta-analysis in Han Chinese. A meta-analysis was piloted in general population by $\mathrm{Xu} \mathrm{H}$ and colleagues [60], which can conclude whether ApoE gene polymorphism prone or protects a subject to Acute Coronary Disease in Han Chinese. As shown in the table, the sample size of Coronary Artery Disease or Acute Coronary Syndrome (few studies) in Han Chinese. Therefore, one can see that the sample size in all of them is inadequate.

\subsection{Relation between Apolipoprotein E Gene Polymorphism and Major Adverse Cardiovascular Events in Patients with Acute Coronary Syndrome}

Major adverse cardiovascular events (MACE), which are the standard clinical combined endpoints to observe the long term prognosis of treatment or risk factors [69] in cardiovascular medicine. There has been merely one study till now reported regarding ApoE, which was conducted in HanChinese, of Beijing. Approximately, 200 hundred patients with myocardial infarction patients were followed for six months and demonstrated higher MACE in MI patient with ApoE gene isoform [13]. This study might have a new insight into the prognostic effect of ApoE genotype on coronary artery disease, specially, acute coronary syndrome. 
Table 1. List of studies regarding the relationship of ApoE gene polymorphism and CHD in Han Chinese (Modified from "Meta-analysis of apolipoprotein E gene polymorphism and susceptibility of myocardial infarction, Xu et al." [64]).

\begin{tabular}{|c|c|c|c|}
\hline \multicolumn{4}{|c|}{ Previous studies regarding the relationship of ApoE and CAD in Han Chinese populatio } \\
\hline Study Name & Study Year & Cases & Controls \\
\hline Jie et al. & 1989 & & \\
\hline Zhang et al. & 1998 & 96 & 131 \\
\hline Yuan et al. & 1998 & 103 & 100 \\
\hline $\mathrm{Li}$ & & 95 & 46 \\
\hline Wu et al. & 1998 & 114 & 135 \\
\hline Yan et al. & 1999 & 93 & 113 \\
\hline Cao et al. & 1999 & 78 & 85 \\
\hline Jin et al. & 1999 & 50 & 100 \\
\hline Peng et al. & 1999 & 220 & 180 \\
\hline Li et al. & 2000 & 95 & 46 \\
\hline Wang et al. & 2000 & 91 & 105 \\
\hline Zhang et al. & 2001 & 91 & 102 \\
\hline Bai et al. & 2001 & 50 & 47 \\
\hline Wang et al. & 2001 & 54 & 71 \\
\hline Zhang et al. & 2001 & 71 & 69 \\
\hline Pan et al. & 2001 & 100 & 50 \\
\hline Yang et al. & 2001 & 204 & 136 \\
\hline Zhang et al. & 2001 & 91 & 102 \\
\hline Wu et al. & 2002 & 172 & 286 \\
\hline Zhang et al. & 2003 & 60 & 71 \\
\hline $\mathrm{Gu} \quad$ et al. & 2003 & 129 & 90 \\
\hline Yang et al. & 2003 & 84 & 39 \\
\hline Liu et al. & 2003 & & \\
\hline Peng et al. & 2003 & 150 & 157 \\
\hline Cao et al. & 2003 & 37 & 72 \\
\hline Yang et al. & 2003 & 40 & 31 \\
\hline Li et al. & 2003 & 125 & 116 \\
\hline Sun et al. & 2004 & 96 & 113 \\
\hline Yin et al. & 2004 & 105 & 110 \\
\hline Liao et al. & 2004 & 86 & 156 \\
\hline He et al. & 2004 & 60 & 104 \\
\hline Xiao et al. & 2005 & 257 & 224 \\
\hline Xiao et al. & 2005 & 257 & 127 \\
\hline Feng et al. & 2005 & 68 & 70 \\
\hline Zou et al. & 2005 & 89 & 83 \\
\hline Pan et al. & 2005 & & \\
\hline Ou et al. & 2005 & 200 & 100 \\
\hline
\end{tabular}


Continued

\begin{tabular}{cccc}
\hline Zhao et al. & 2006 & 29 & 116 \\
Liang et al. & 2006 & 133 & 122 \\
Ma et al. & 2006 & 88 & 75 \\
Xie et al. & 2006 & 78 & 100 \\
Wang et al. & 2006 & 201 & 360 \\
Xu. et al. & 2007 & 52 & 60 \\
Chu et al. & 2007 & 329 & 220 \\
Wang et al. & 2007 & & \\
Ding et al. & 2008 & 52 & 110 \\
Ma et al. & 2011 & 88 & 75 \\
\hline
\end{tabular}

\subsection{Mechanism of ApoE Allele in Increasing the Risk of Coronary Artery Disease}

There are some proposed mechanisms, which can lead to Coronary Artery Atherosclerotic and subsequent Coronary Artery Disease but the lipid pathway is the most discussed of all is the pathways.

It is assumed that ApoE4 phenotype has extra affinity to the receptor on the hepatic cells. However, as a negative feedback, the number of receptors decrease, subsequently causing dyslipidemia and atherosclerotic plaque. The cap of the atherosclerotic plaque denudes and Acute Coronary Syndrome occurs (Figure 6).

On the other hand, people with ApoE2 allele are known to be protective in terms of cardiovascular diseases. In contrast to ApoE E 4, ApoE E2 has lower affinity for its receptor, which in turn causes increased number of receptors, thus leading to decreased level of plasma lipid. Thus, ApoE2 carriers are shielding to cardiovascular diseases.

Similarly, it was revealed by Cenarro A et al. in their case control study on autosomal dominant hypercholesteremic patients, showed that $3.1 \%$ patients, who had no mutation ofLDLR, APOB, and PCSK9 mutations but mutation in ApoE gene. This mutated ApoE E protein leads to decreased numbers of receptors, which consequently primed to hypercholesterolemia [70]. From the animals studies it has been shown that those animals which were deficient in ApoE E, after three to four months of follow-up with chow-fed diet, developed atherosclerotic lesions in major systemic and pulmonary arteries [35] [71].

Alternatively, the rate of atherosclerotic lesions speeded in ApoE E knocked out mice fed with western diet. This verifies that ApoE E plays an important role in the pathogenesis of cardiovascular diseases. In addition, life style and diet have additive effect. There are no studies yet done on how much a person with particular allele of ApoE E contributes to the Coronary Artery Disease.

\section{Can ApoE E Genotype Be Used as a Screening Test for Cardiovascular Diseases?}

Since Apolipoprotein E phenotype has an essential contributor in the metabolism 


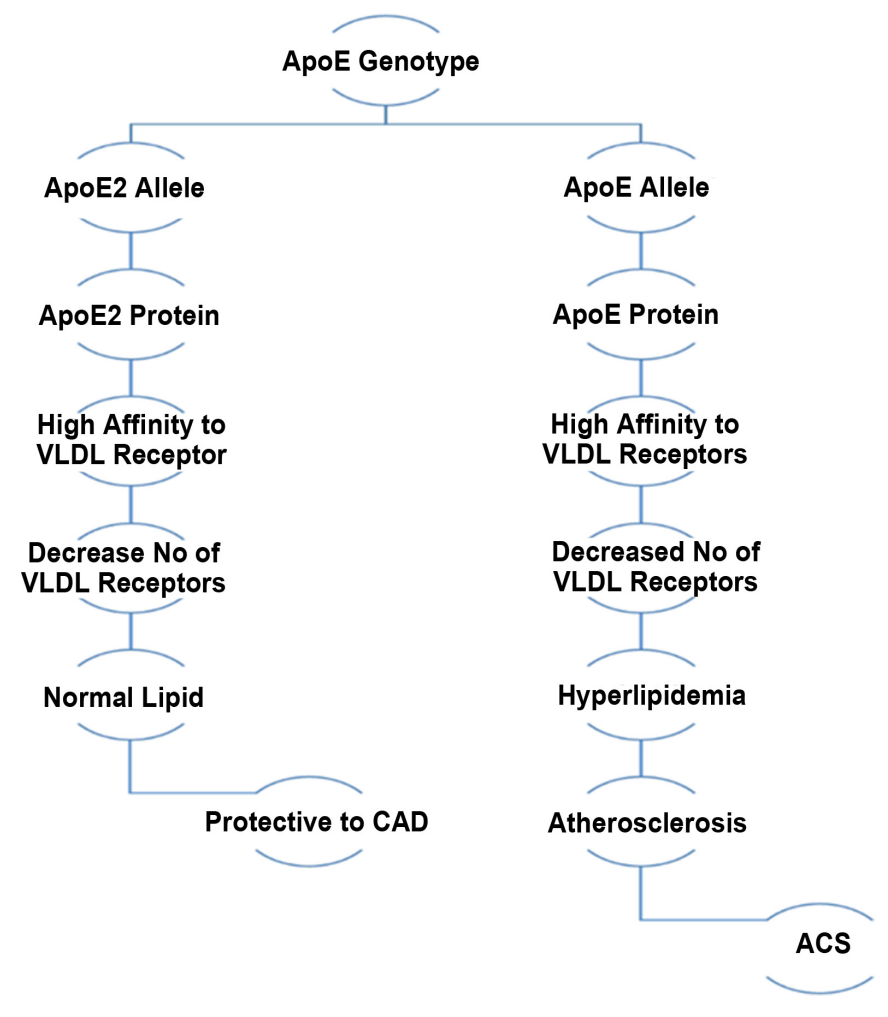

Figure 6. Illustrates the mechanism of ApoE genotype causing Coronary Artery Disease and in turn Acute Coronary Syndrome.

of any alteration in the phenotype would change both the blood lipid level and also subsequently alter the therapeutic effect of anti-lipid therapy. Now, the question is, "Can we test ApoE E gene as a screening test for healthy adults and predict the risk ratio for Coronary Artery Disease?" In addition, "Can we use test ApoE E genotype before starting anti-hyperlipidemic medication in healthy people to foreshadow the efficacy of the medication in particular those patients who are resistant to Statin?" considering the cost effect of ApoE tests. In China, ApoE test costs about 700 Chinese Yuan, which is almost equal to 120 US dollars. It would be still naïve to test individual for ApoE E genotype with such high price, which does not affect the treatment plan or one cannot yet propose any intervention with indemnity. Hence, it is prerequisite to conduct a prospective study on normal healthy adults and follow them prospectively.

\section{Conclusions}

In inference, although ApoE gene polymorphism might be one of the strong genetic factors contributing in the pathogenesis of hyperlipidemia, and response to Statin and most importantly in the pathogenesis of Coronary Artery Disease, especially Acute Coronary Syndrome in Han Chinese population like other populations, yet one requires to conduct a prospective study with bigger sample size in Han Chinese population.

The studies verify that ApoE4 carrier is a genetic risk factor in Han Chinese like most of the other populations while ApoE2 carrier is a genetic protective 
factor. In addition, ApoE4 carrier also has higher incidence of MACE and even severe stenosis of coronary artery. However, it is still premature to agree that every patient with ACS or taking Statin for Coronary Artery Disease should be priorly tested for ApoE gene because it is not confirmed whether ApoE will assist in intervention or modify the therapeutic plan.

Despite all these reports, this zone of research still requires extensive investigations and prospective studies containing enormous size to demonstrate decisive judgment pertaining relationship of ApoE gene polymorphism with Acute Coronary Syndrome in Han Chinese population.

\section{Conflicts of Interest}

The authors declare no conflicts of interest regarding the publication of this paper.

\section{References}

[1] Zhang, X., Lu, Z. and Liu, L. (2008) Coronary Heart Disease in China. Heart, 94, 1126-1131. https://doi.org/10.1136/hrt.2007.132423

[2] Hu, S. and Kong, L. (2006) Report on Cardiovascular Diseases in China. Encyclopedia of China Publishing House, Beijing, 85.

[3] Chen, W.W., Gao, R.L., Liu, L.S., Zhu, M.L., Wang, W., Wang, Y.J., et al. (2017) China Cardiovascular Diseases Report 2015: A Summary. Journal of Geriatric Cardiology, 14, 1-10.

[4] Institute of Medicine Committee on Preventing the Global Epidemic of Cardiovascular Disease (2010) Meeting the Challenges in Developing C. The National Academies Collection: Reports funded by National Institutes of Health. In: Fuster, V. and Kelly, B.B., Eds., Promoting Cardiovascular Health in the Developing World: $A$ Critical Challenge to Achieve Global Health, National Academies Press (US) National Academy of Sciences, Washington DC.

[5] Sahin, S., Ceyhan, K., Benli, I., Ozyurt, H., Naseri, E., Tumuklu, M.M., et al. (2015) Traditional Risk Factors and Angiotensin-Converting Enzyme Insertion/Deletion Gene Polymorphism in Coronary Artery Disease. Genetics and Molecular Research, 14, 2063-2068. https://doi.org/10.4238/2015.March.20.16

[6] Wilson, P.W. (1994) Established Risk Factors and Coronary Artery Disease: The Framingham Study. American Journal of Hypertension, 7, 7S-12S. https://doi.org/10.1093/ajh/7.7.7S

[7] Wilson, P.W. (2008) Progressing from Risk Factors to Omics. Circulation: Cardiovascular Genetics, 1, 141-146. https://doi.org/10.1161/CIRCGENETICS.108.815605

[8] Damani, S.B. and Topol, E.J. (2007) Future Use of Genomics in Coronary Artery Disease. Journal of the American College of Cardiology, 50, 1933-1940. https://doi.org/10.1016/j.jacc.2007.07.062

[9] Pang, J., Poulter, E.B., Bell, D.A., Bates, T.R., Jefferson, V.L., Hillis, G.S., et al. (2015) Frequency of Familial Hypercholesterolemia in Patients with Early-Onset Coronary Artery Disease Admitted to a Coronary Care Unit. Journal of Clinical Lipidology, 9 , 703-708. https://doi.org/10.1016/j.jacl.2015.07.005

[10] Mahley, R.W. (1988) Apolipoprotein E: Cholesterol Transport Protein with Expanding Role in Cell Biology. Science, 240, 622-630. 
https://doi.org/10.1126/science.3283935

[11] Scott, J., Knott, T.J., Shaw, D.J. and Brook, J.D. (1985) Localization of Genes Encoding Apolipoproteins CI, CII, and E to the p13 $\rightarrow$ Cen Region of Human Chromosome 19. Human Genetics, 71, 144-146. https://doi.org/10.1007/BF00283370

[12] Huebbe, P. and Rimbach, G. (2017) Evolution of Human Apolipoprotein E (APOE) Isoforms: Gene Structure, Protein Function and Interaction with Dietary Factors. Ageing Research Reviews, 37, 146-161. https://doi.org/10.1016/j.arr.2017.06.002

[13] Xia, J., Hu, S., Yin, C. and Xu, D. (2018) A 6-Month Follow-Up Study of the Relation between Apolipoprotein E Gene Polymorphism and Major Adverse Cardiovascular Events in Patients with Acute Coronary Syndrome. Cardiology, 140, 187-193. https://doi.org/10.1159/000491597

[14] Mahley, R.W. and Rall Jr., S.C. (2000) Apolipoprotein E: Far More Than a Lipid Transport Protein. Annual Review of Genomics and Human Genetics, 1, 507-537. https://doi.org/10.1146/annurev.genom.1.1.507

[15] Song, Y., Stampfer, M.J. and Liu, S. (2004) Meta-Analysis: Apolipoprotein E Genotypes and Risk for Coronary Heart Disease. Annals of Internal Medicine, 141, 137-147. https://doi.org/10.7326/0003-4819-141-2-200407200-00013

[16] Larifla, L., Armand, C., Bangou, J., Blanchet-Deverly, A., Numeric, P., Fonteau, C., et al. (2017) Association of APOE Gene Polymorphism with Lipid Profile and Coronary Artery Disease in Afro-Caribbeans. PLoS ONE, 12, e0181620. https://doi.org/10.1371/journal.pone.0181620

[17] Corbo, R.M. and Scacchi, R. (1999) Apolipoprotein E (APOE) Allele Distribution in the World. Is $A P O E^{\star} 4$ a 'Thrifty' Allele? Annals of Human Genetics, 63, 301-310. https://doi.org/10.1046/j.1469-1809.1999.6340301.x

[18] Wetterau, J.R., Aggerbeck, L.P., Rall Jr., S.C. and Weisgraber, K.H. (1988) Human Apolipoprotein E3 in Aqueous Solution. I. Evidence for Two Structural Domains. The Journal of Biological Chemistry, 263, 6240-6248.

[19] Saito, H., Dhanasekaran, P., Nguyen, D., Baldwin, F., Weisgraber, K.H., Wehrli, S., et al. (2003) Characterization of the Heparin Binding Sites in Human Apolipoprotein E. The Journal of Biological Chemistry, 278, 14782-14787. https://doi.org/10.1074/jbc.M213207200

[20] Phillips, M.C. (2014) Apolipoprotein E Isoforms and Lipoprotein Metabolism. IUBMB Life, 66, 616-623. https://doi.org/10.1002/iub.1314

[21] Mahley, R.W. and Ji, Z.-S. (1999) Remnant Lipoprotein Metabolism: Key Pathways Involving Cell-Surface Heparan Sulfate Proteoglycans and Apolipoprotein E. Journal of Lipid Research, 40, 1-16.

[22] Herz, J., Chen, Y., Masiulis, I. and Zhou, L. (2009) Expanding Functions of Lipoprotein Receptors. Journal of Lipid Research, 50, S287-S92. https://doi.org/10.1194/jlr.R800077-JLR200

[23] Khoo, C., Campos, H., Judge, H. and Sacks, F.M. (1999) Effects of Estrogenic Oral Contraceptives on the Lipoprotein B Particle System Defined by Apolipoproteins E and C-III Content. Journal of Lipid Research, 40, 202-212.

[24] Dallongeville, J., Bauge, E., Lebel, P. and Fruchart, J.C. (1997) Fat Ingestion Is Associated with Increased Levels of ApoC-III- and ApoE-B-Containing Lipoprotein Particles in Humans. European Journal of Clinical Investigation, 27, 1055-1060. https://doi.org/10.1046/j.1365-2362.1997.2350782.x

[25] Boehm-Cagan, A., Bar, R., Liraz, O., Bielicki, J.K., Johansson, J.O. and Michaelson, D.M. (2016) ABCA1 Agonist Reverses the ApoE4-Driven Cognitive and Brain Pa- 
thologies. Journal of Alzheimer's Disease, 54, 1219-1233.

https://doi.org/10.3233/JAD-160467

[26] Weintraub, M.S., Eisenberg, S. and Breslow, J.L. (1987) Dietary Fat Clearance in Normal Subjects Is Regulated by Genetic Variation in Apolipoprotein E. The Jour nal of Clinical Investigation, 80, 1571-1577. https://doi.org/10.1172/JCI113243

[27] Bioletto, S., Fontana, P., Darioli, R. and James, R.W. (1998) Apolipoprotein E Polymorphism and the Distribution Profile of Very Low Density Lipoproteins: An Influence of the E4 Allele on Large (Sf > 60) Particles. Atherosclerosis, 138, 207-215. https://doi.org/10.1016/S0021-9150(98)00002-1

[28] Chaefer, E.J., Lamon-Fava, S., Johnson, S., Ordovas, J.M., Schaefer, M.M., Castelli, W.P., et al. (1994) Effects of Gender and Menopausal Status on the Association of Apolipoprotein E Phenotype with Plasma Lipoprotein Levels. Results from the Framingham Offspring Study. Arteriosclerosis and Thrombosis. A Journal of Vascular Biology, 14, 1105-1113. https://doi.org/10.1161/01.ATV.14.7.1105

[29] Dart, A.M. and Cooper, B. (1999) Independent Effects of Apo E Phenotype and Plasma Triglyceride on Lipoprotein Particle Sizes in the Fasting and Postprandial States. Arteriosclerosis, Thrombosis, and Vascular Biology, 19, 2465-2473. https://doi.org/10.1161/01.ATV.19.10.2465

[30] Topic, A., Spasojevic Kalimanovska, V., Zeljkovic, A., Vekic, J. and Jelic Ivanovic, Z. (2008) Gender-Related Effect of Apo E Polymorphism on Lipoprotein Particle Sizes in the Middle-Aged Subjects. Clinical Biochemistry, 41, 361-367. https://doi.org/10.1016/j.clinbiochem.2007.11.013

[31] Akanji, A.O., Suresh, C.G., Fatania, H.R., Al-Radwan, R. and Zubaid, M. (2007) Associations of Apolipoprotein E Polymorphism with Low-Density Lipoprotein Size and Subfraction Profiles in Arab Patients with Coronary Heart Disease. Metabolism: Clinical and Experimental, 56, 484-490. https://doi.org/10.1016/j.metabol.2006.11.006

[32] Bach-Ngohou, K., Giraud, F., Krempf, M. and Bard, J.M. (2001) Influence of Remnant Accumulation Markers on Plasma Concentrations of Two Lipoprotein(a) Subspecies (Containing or Free of ApoE). Metabolism: Clinical and Experimental, 50, 277-282. https://doi.org/10.1053/meta.2001.21031

[33] Moriarty, P.M., Varvel, S.A., Gordts, P.L., McConnell, J.P. and Tsimikas, S. (2017) Lipoprotein(a) Mass Levels Increase Significantly According to APOE Genotype: An Analysis of 431239 Patients. Arteriosclerosis, Thrombosis, and Vascular Biology, 37, 580-588. https://doi.org/10.1161/ATVBAHA.116.308704

[34] Moreno, J.A., Perez-Jimenez, F., Marin, C., Gomez, P., Perez-Martinez, P., Moreno, R., et al. (2004) The Effect of Dietary Fat on LDL Size Is Influenced by Apolipoprotein E Genotype in Healthy Subjects. The Journal of Nutrition, 134, 2517-2522. https://doi.org/10.1093/jn/134.10.2517

[35] Zhang, S.H., Reddick, R.L., Piedrahita, J.A. and Maeda, N. (1992) Spontaneous Hypercholesterolemia and Arterial Lesions in Mice Lacking Apolipoprotein E. Science, 258, 468-471. https://doi.org/10.1126/science.1411543

[36] Lu, Z., Wu, X., Jin, X., Peng, F. and Lin, J. (2016) Apolipoprotein E $\varepsilon 2 / \varepsilon 3 / \varepsilon 4$ Variant in Association with Obstructive Sleep Apnoea and Lipid Profile: A Meta-Analysis. The Journal of International Medical Research, 44, 3-14. https://doi.org/10.1177/0300060515611539

[37] Al Harthi, F., Huraib, G.B., Zouman, A., Arfin, M., Tariq, M. and Al-Asmari, A. (2014) Apolipoprotein E Gene Polymorphism and Serum Lipid Profile in Saudi Patients with Psoriasis. Disease Markers, 2014, Article ID: 239645. 
https://doi.org/10.1155/2014/239645

[38] El-Lebedy, D., Raslan, H.M. and Mohammed, A.M. (2016) Apolipoprotein E Gene Polymorphism and Risk of Type 2 Diabetes and Cardiovascular Disease. Cardiovascular Diabetology, 15, 12. https://doi.org/10.1186/s12933-016-0329-1

[39] Procopciuc, L.M., Caracostea, G., Zaharie, G. and Stamatian, F. (2015) Newborn APOE Genotype Influences Maternal Lipid Profile and the Severity of High-Risk Pregnancy-Preeclampsia: Interaction with Maternal Genotypes as a Modulating Risk Factor in Preeclampsia. Hypertension in Pregnancy, 34, 271-283. https://doi.org/10.3109/10641955.2015.1009541

[40] Boulenouar, H., Mediene Benchekor, S., Meroufel, D.N., Lardjam Hetraf, S.A., Ouhaibi Djellouli, H., Hermant, X., et al. (2013) Impact of APOE Gene Polymorphisms on the Lipid Profile in an Algerian Population. Lipids in Health and Disease, 12, 155. https://doi.org/10.1186/1476-511X-12-155

[41] Versmissen, J., Oosterveer, D.M., Hoekstra, M., Out, R., Berbee, J.F., Blommesteijn-Touw, A.C., et al. (2011) Apolipoprotein Isoform E4 Does Not Increase Coronary Heart Disease Risk in Carriers of Low-Density Lipoprotein Receptor Mutations. Circulation Cardiovascular Genetics, 4, 655-660. https://doi.org/10.1161/CIRCGENETICS.111.959858

[42] Gan, W., Liu, Y., Luo, K.H., Liang, S.S., Wang, H., Li, M., et al. (2018) The Prevalence Change of Hyperlipidemia and Hyperglycemia and the Effectiveness of Yearly Physical Examinations: An Eight-Year Study in Southwest China. Lipids in Health and Disease, 17, 70. https://doi.org/10.1186/s12944-018-0724-6

[43] Xiang, R., Fan, L.L., Lin, M.J., Li, J.J., Shi, X.Y., Jin, J.Y., et al. (2017) The Genetic Spectrum of Familial Hypercholesterolemia in the Central South Region of China. Atherosclerosis, 258, 84-88. https://doi.org/10.1016/j.atherosclerosis.2017.02.007

[44] Xu, X., Li, J., Sheng, W. and Liu, L. (2008) Meta-Analysis of Genetic Studies from Journals Published in China of Ischemic Stroke in the Han Chinese Population. $\mathrm{Ce}$ rebrovascular Diseases, 26, 48-62. https://doi.org/10.1159/000135653

[45] Corsetti, J.P., Sparks, C.E., Bakker, S.J.L., Gruppen, E.G. and Dullaart, R.P.F. (2018) Roles of High Apolipoprotein E Blood Levels and HDL in Development of Familial Dysbetalipoproteinemia in $\varepsilon 2 \varepsilon 2$ Subjects. Clinical Biochemistry, 52, 67-72. https://doi.org/10.1016/j.clinbiochem.2017.11.010

[46] Kitahara, M., Shinomiya, M., Shirai, K., Saito, Y. and Yoshida, S. (1990) Frequency and Role of Apo E Phenotype in Familial Hypercholesterolemia and Non-Familial Hyperlipidemia in the Japanese. Atherosclerosis, 82, 197-204.

https://doi.org/10.1016/0021-9150(90)90041-G

[47] Xia, Y., Sass, C., Shen, X., Siest, G. and Visvikis, S. (2000) Associations of Apolipoprotein E Concentration and Polymorphism with Lipids and Apolipoprotein Levels in Chinese from Beijing and Shanghai. Clinical Chemistry and Laboratory Medicine, 38, 655-659. https://doi.org/10.1515/CCLM.2000.094

[48] Kajinami, K., Takekoshi, N., Brousseau, M.E. and Schaefer, E.J. (2004) Pharmacogenetics of HMG-CoA Reductase Inhibitors: Exploring the Potential for Genotype-Based Individualization of Coronary Heart Disease Management. Atherosclerosis, 177, 219-234. https://doi.org/10.1016/j.atherosclerosis.2004.09.004

[49] Mangravite, L.M., Thorn, C.F. and Krauss, R.M. (2006) Clinical Implications of Pharmacogenomics of Statin Treatment. The Pharmacogenomics Journal, 6, 360-374. https://doi.org/10.1038/sj.tpj.6500384

[50] Banach, M., Stulc, T., Dent, R. and Toth, P.P. (2016) Statin Non-Adherence and Residual Cardiovascular Risk: There Is Need for Substantial Improvement. Interna- 
tional Journal of Cardiology, 225, 184-196.

https://doi.org/10.1016/j.ijcard.2016.09.075

[51] Zintzaras, E., Kitsios, G.D., Triposkiadis, F., Lau, J. and Raman, G. (2009) APOE Gene Polymorphisms and Response to Statin Therapy. The Pharmacogenomics Journal, 9, 248-257. https://doi.org/10.1038/tpj.2009.25

[52] Kirac, D., Bayam, E., Dagdelen, M., Gezmis, H., Sarikaya, S., Pala, S., et al. (2017) HMGCR and ApoE Mutations May Cause Different Responses to Lipid Lowering Statin Therapy. Cellular and Molecular Biology, 63, 43-48. https://doi.org/10.14715/cmb/2017.63.10.6

[53] Hu, M., Mak, V.W. and Tomlinson, B. (2012) Polymorphisms in Apolipoprotein E and Apolipoprotein A-V Do Not Influence the Lipid Response to Rosuvastatin but Are Associated with Baseline Lipid Levels in Chinese Patients with Hyperlipidemia. Journal of Clinical Lipidology, 6, 585-592. https://doi.org/10.1016/j.jacl.2012.02.005

[54] Zhang, Y., Wei, D.D., Yuan, R.R., Ge, Q.X., Chen, F., Yang, S.X., et al. (2017) Effects of ApoE Gene Polymorphism on the Efficacy of Atorvastatin in the Treatment of Hyperlipidemia. National Medical Journal of China, 97, 291-294.

[55] Tavintharan, S., Lim, S.C., Chan, Y.H. and Sum, C.F. (2007) Apolipoprotein E Genotype Affects the Response to Lipid-Lowering Therapy in Chinese Patients with Type 2 Diabetes Mellitus. Diabetes, Obesity \& Metabolism, 9, 81-86. https://doi.org/10.1111/j.1463-1326.2006.00577.x

[56] Mahley, R.W., Weisgraber, K.H. and Huang, Y. (2009) Apolipoprotein E: Structure Determines Function, from Atherosclerosis to Alzheimer's Disease to AIDS. The Journal of Lipid Research, 50, S183-S188. https://doi.org/10.1194/jlr.R800069-JLR200

[57] Kritharides, L., Nordestgaard, B.G., Tybjaerg-Hansen, A., Kamstrup, P.R. and Afzal, S. (2017) Effect of $A P O E \varepsilon$ Genotype on Lipoprotein(a) and the Associated Risk of Myocardial Infarction and Aortic Valve Stenosis. The Journal of Clinical Endocrinology and Metabolism, 102, 3390-3399. https://doi.org/10.1210/jc.2017-01049

[58] Tyynela, P., Goebeler, S., Ilveskoski, E., Mikkelsson, J., Perola, M., Lehtimaki, T., et al. (2013) Age-Dependent Interaction of Apolipoprotein E Gene with Eastern Birthplace in Finland Affects Severity of Coronary Atherosclerosis and Risk of Fatal Myocardial Infarction-Helsinki Sudden Death Study. Annals of Medicine, 45, 213-219. https://doi.org/10.3109/07853890.2012.727021

[59] Tanguturi, P., Pullareddy, B., Kumar, P.S. and Murthy, D.K. (2013) Association between Apolipoprotein E Gene Polymorphism and Myocardial Infarction. Biochemical Genetics, 51, 398-405. https://doi.org/10.1007/s10528-013-9572-2

[60] Xu, H., Li, H., Liu, J., Zhu, D., Wang, Z., Chen, A., et al. (2014) Meta-Analysis of Apolipoprotein E Gene Polymorphism and Susceptibility of Myocardial Infarction. PLoS ONE, 9, e104608. https://doi.org/10.1371/journal.pone.0104608

[61] Ranjith, N., Pegoraro, R.J., Rom, L., Rajput, M.C. and Naidoo, D.P. (2004) Lp(a) and ApoE Polymorphisms in Young South African Indians with Myocardial Infarction. Cardiovascular Journal of South Africa: Official Journal for Southern Africa Cardiac Society and South African Society of Cardiac Practitioners, 15, 111-117.

[62] Zhao, Q.R., Lei, Y.Y., Li, J., Jiang, N. and Shi, J.P. (2017) Association between Apolipoprotein E Polymorphisms and Premature Coronary Artery Disease: A Meta-Analysis. Clinical Chemistry and Laboratory Medicine, 55, 284-298. https://doi.org/10.1515/cclm-2016-0145

[63] Xu, S., Yin, X., Li, S., Jin, W., Lou, H., Yang, L., et al. (2009) Genomic Dissection of 
Population Substructure of Han Chinese and Its Implication in Association Studies. The American Journal of Human Genetics, 85, 762-774. https://doi.org/10.1016/j.ajhg.2009.10.015

[64] Zhang, M.D., Gu, W., Qiao, S.B., Zhu, E.J., Zhao, Q.M. and Lv, S.Z. (2014) Apolipoprotein E Gene Polymorphism and Risk for Coronary Heart Disease in the Chinese Population: A Meta-Analysis of 61 Studies Including 6634 Cases and 6393 Controls. PLoS ONE, 9, e95463. https://doi.org/10.1371/journal.pone.0095463

[65] Liang, S., Pan, M., Geng, H.H., Chen, H., Gu, L.Q., Qin, X.T., et al. (2009) Apolipoprotein E Polymorphism in Normal Han Chinese Population: Frequency and Effect on Lipid Parameters. Molecular Biology Reports, 36, 1251-1256. https://doi.org/10.1007/s11033-008-9305-5

[66] Yin, Y.W., Sun, Q.Q., Zhang, B.B., Hu, A.M., Liu, H.L., Wang, Q., et al. (2013) Association between Apolipoprotein E Gene Polymorphism and the Risk of Coronary Artery Disease in Chinese Population: Evidence from a Meta-Analysis of 40 Studies. PLoS ONE, 8, e66924. https://doi.org/10.1371/journal.pone.0066924

[67] Bai, X., Zhao, M. and Wang, B. (2001) Dyslipidemia-Related Risk Factors for Myocardial Infarction and Polymorphism of ApoE Gene among Myocardial Infarction Patients and Their Siblings. National Medical Journal of China, 81, 340-343.

[68] Baum, L., Ng, H.K., Wong, K.S., Tomlinson, B., Rainer, T.H., Chen, X., et al. (2006) Associations of Apolipoprotein E Exon 4 and Lipoprotein Lipase S447X Polymorphisms with Acute Ischemic Stroke and Myocardial Infarction. Clinical Chemistry and Laboratory Medicine, 44, 274-281. https://doi.org/10.1515/CCLM.2006.047

[69] Kip, K.E., Hollabaugh, K., Marroquin, O.C. and Williams, D.O. (2008) The Problem with Composite End Points in Cardiovascular Studies: The Story of Major Adverse Cardiac Events and Percutaneous Coronary Intervention. Journal of the American College of Cardiology, 51, 701-707. https://doi.org/10.1016/j.jacc.2007.10.034

[70] Cenarro, A., Etxebarria, A., de Castro-Oros, I., Stef, M., Bea, A.M., Palacios, L., et al. (2016) The p.Leu167del Mutation in APOE Gene Causes Autosomal Dominant Hypercholesterolemia by Down-Regulation of LDL Receptor Expression in Hepatocytes. The Journal of Clinical Endocrinology and Metabolism, 101, 2113-2121. https://doi.org/10.1210/jc.2015-3874

[71] Plump, A.S., Smith, J.D., Hayek, T., Aalto-Setala, K., Walsh, A., Verstuyft, J.G., et al. (1992) Severe Hypercholesterolemia and Atherosclerosis in Apolipoprotein E-Deficient Mice Created by Homologous Recombination in ES Cells. Cell, 71, 343-353. https://doi.org/10.1016/0092-8674(92)90362-G 